\title{
CORRELAÇÃO LINEAR E ESPACIAL ENTRE PRODUTIVIDADE DE BRACHIARIA BRIZANTHA, DENSIDADE DO SOLO E POROSIDADE TOTAL EM FUNÇÃO DO SISTEMA DE MANEJO DO SOLO
}

\author{
EDNEY L. DA VITÓRIA ${ }^{1}$, HAROLDO C. FERNANDES ${ }^{2}$, MAURI M. TEIXEIRA ${ }^{3}$, \\ PAULO R. CECON ${ }^{4}$, ELCIO DAS G. LACERDA ${ }^{5}$
}

\begin{abstract}
RESUMO: A densidade e a porosidade são propriedades físicas do solo que são alteradas em função do sistema de manejo utilizado, com consequente influência sobre a produtividade das culturas. A produtividade da planta forrageira, Brachiaria brizantha, em função da densidade do solo e da porosidade total foi analisada em dois sistemas de manejo de solo, em experimento conduzido no segundo semestre de 2009, no município de Santa Teresa, no Estado do Espírito Santo. O objetivo foi estudar a variabilidade e as correlações lineares e espaciais entre os atributos da planta e do solo, visando a selecionar um indicador da qualidade física do solo de boa representatividade para produtividade de forragem. Marcaram-se duas parcelas de $40 \mathrm{~m}$ por $50 \mathrm{~m}$ a cada $5 \mathrm{~m}$, em duas direções, resultando em um reticulado retangular de 99 pontos, em cada um dos sistemas utilizados: preparo convencional e plantio direto. Os atributos estudados, além de não terem variado aleatoriamente, apresentaram variabilidade dos dados entre média e alta, e seguiram padrões espaciais bem definidos, com alcance entre 20,3 e 24,2 m. Por sua vez, a correlação linear entre o atributo da planta e os do solo, em função do elevado número de observações, foi baixa. A melhor correlação para produtividade de matéria seca foi com a densidade do solo na profundidade de $0,0-0,15 \mathrm{~m}$, independentemente do sistema de manejo do solo, indicando que a produtividade e a densidade do solo são inversamente proporcionais. Portanto, a densidade do solo avaliada na camada de $0,0-0,15 \mathrm{~m}$ apresentou-se como satisfatório indicador da qualidade física do solo, quando se considerou a produtividade da forrageira.
\end{abstract}

PALAVRAS-CHAVE: propriedades físicas do solo, geoestatística, preparo do solo, forragicultura.

\section{LINEAR AND SPATIAL CORRELATIONS AMONG FORAGE YIELD, BULK DENSITY AND TOTAL POROSITY IN FUNCTION OF SOIL MANAGEMENT SYSTEM}

\begin{abstract}
Total porosity and bulk density are strongly affected by soil management, which reflects directly in agricultural productivity. The productivity of the forage, Brachiaria brizantha, in function of bulk density and total porosity was analyzed in two soil management systems, in an experiment lead in the second semester of 2009, in Santa Teresa County, state of Espírito Santo, Brazil. The purpose of the study was to evaluate the variability, and linear and spatial correlations among the attributes (plant and soil) in order to identify an indicator of soil physical quality for corn forage productivity. A geostatistical grid was installed to collect soil and plant data, with 99 sample points, in each soil management system. The studied attributes did not vary randomly and the variability was medium to low, with well defined patterns. The spatial range varied between 20.3 and $24.2 \mathrm{~m}$. On the other hand, the linear correlations between the forage productivity with the soil attributes were low due to the high data number. The comments of better correlation for productivity, was the bulk density in the depth of $0.0-0.15$ independently of the soil management system, indicating that the productivity and the density of the soil are inversely proportional. Thus, the bulk density in the $0.0-0.15 \mathrm{~m}$ soil layer proved to be a satisfactory index of the soil physical quality regarding to forage yield.
\end{abstract}

KEYWORDS: soil physical attributes, geostatistics, soil management, crop forage.

\footnotetext{
${ }^{1}$ Eng ${ }^{0}$ Agrícola, Prof. Adjunto, Departamento de Ciências Agrárias e Biológicas, UFES, Câmpus São Mateus - ES, edney.vitoria@ceunes.ufes.br.

${ }^{2}$ Engo Agrícola, Prof. Associado, Departamento de Engenharia Agrícola, UFV, Viçosa - MG, haroldo@ufv.br.

${ }^{3}$ Engo Agrônomo, Prof. Associado, Departamento de Engenharia Agrícola, UFV, Viçosa - MG, mauri@ufv.br.

${ }^{4}$ Eng $^{\mathrm{o}}$ Agrônomo, Prof. Associado, Departamento de Estatística, UFV, Viçosa - MG, cecon@ufv.br.

${ }^{5}$ Tecnólogo em Agropecuária, Prof. Voluntário, IFES, Câmpus Santa Teresa - ES.

Recebido pelo Conselho Editorial em: 5-7-2011
}

Aprovado pelo Conselho Editorial em: 29-5-2012 


\section{INTRODUÇÃO}

As espécies forrageiras representam as plantas de interesse econômico mais cultivadas no Brasil e no mundo. Contudo, o pequeno número de espécies forrageiras com valor nutricional satisfatório, aliado à baixa fertilidade e manejo inadequado do solo, constitui um dos principais fatores limitantes da produção pecuária nas regiões tropicais.

A intensificação da mecanização é usada constantemente com intuito de melhorar as condições de implantação e desenvolvimento de plantas forrageiras. Entretanto, muitas vezes, a produtividade é comprometida pelo excesso ou pela inadequação de práticas às quais o solo é submetido, desde o seu manejo até à colheita da cultura que nele se estabeleceu.

A distribuição espacial de propriedades de solo e de produtividade tem recebido atenção crescente por parte de pesquisadores de vários países e em muitas áreas do conhecimento. Estudos de solos foram os primeiros a serem realizados, evidenciando a influência da distância amostral em contraposição à crença vigente de aleatoriedade espacial. A variabilidade espacial das propriedades do solo tem sido objeto de estudo desde que os pesquisadores passaram a observar a pouca eficácia das ferramentas clássicas da estatística em estudos de solos, e é justamente nestes casos que a geoestatística tem suas principais aplicações.

Em termos de variáveis físicas, o trabalho de LIMA et al. (2009a) envolve o estudo das variações de porosidade e densidade do solo em manejos diferentes do solo. FOLONI et al. (2003), SECCO et al. (2005) e FREDDI (2007) mostram a correlação espacial entre produtividade de forrageiras e variáveis de solo, destacando-se como os principais estudos envolvendo variáveis de solo e de produtividade.

A produtividade vegetal depende de vários fatores, dentre os quais a densidade e a porosidade do solo. A afirmação foi constatada por SANTOS et al. (2006) ao estudarem a produtividade de grãos de milho, irrigado com pivô central, as densidades da partícula e do solo e a porosidade total de um Latossolo Vermelho distroférrico, sob plantio direto, com o objetivo de estudar as correlações lineares e espaciais entre os atributos. Os atributos do solo apresentaram baixa variabilidade de seus dados, sendo média no da planta, concluindo-se que entre os atributos do solo, as correlações lineares simples variaram elasticamente. Contudo, quando correlacionados com a produtividade, tais correlações pouco variaram. Ainda que com reservas devido à baixa correlação, com o aumento da densidade do solo ocorreu uma diminuição da produtividade.

O sistema de manejo do solo é considerado uma das fontes mais importantes de variabilidade das propriedades físicas do solo que, por sua vez, podem influenciar sobre a produtividade de culturas. E alguns trabalhos têm comparado sistemas de manejo com e sem revolvimento do solo (STONE \& SILVEIRA, 2001). Neste contexto, a hipótese deste estudo é de que a utilização do preparo convencional e o plantio direto determinam a amplitude da dependência espacial das propriedades físicas, em consequência, a variabilidade da produtividade de matéria seca e o índice de área foliar da braquiária. Para confirmar ou negar esta hipótese, os objetivos deste estudo são: (1) estudar a distribuição dos dados e ajustá-los às exigências das ferramentas geoestatísticas; (2) estabelecer e descrever a estrutura de dependência espacial de cada variável, por meio dos semivariogramas, usando a validação cruzada para escolher o melhor modelo de estimativa por krigagem; (3) gerar mapas de contorno de isolinhas para todas as variáveis com dependência espacial, e (4) descrever a correlação espacial entre as variáveis que apresentarem maior correlação espacial, duas a duas, por meio dos semivariogramas cruzados.

\section{MATERIAL E MÉTODOS}

Este trabalho foi realizado na área experimental do Instituto Federal de Ensino Superior, Câmpus de Santa Teresa, localizada no município de Santa Teresa, região Serrana do Espírito Santo, situado entre as coordenadas 19049'09' de latitude sul e 40 $40^{\prime} 17^{\prime \prime}$ de longitude oeste de Greenwich, e altitude média de $675 \mathrm{~m}$. O clima da região é temperado úmido com inverno seco e 
verão quente. A média de temperatura anual entre 16 e $18{ }^{\circ} \mathrm{C}$. O solo é classificado como Latossolo Amarelo eutrófico, textura arenosa, com $300 \mathrm{~g} \mathrm{~kg}^{-1}$ de argila, $70 \mathrm{~g} \mathrm{~kg}^{-1}$ de silte e $630 \mathrm{~g} \mathrm{~kg}^{-1}$ de areia, conforme NEIRO (2002).

A produtividade da planta forrageira, Brachiaria brizantha, em função da densidade do solo e da porosidade total, foi analisada em dois sistemas de manejo de solo. A densidade do solo foi determinada utilizando a seguinte expressão:

$$
\mathrm{Ds}=\frac{\mathrm{Ms}}{\mathrm{Va}}
$$

em que,

Ds - densidade do solo, $\mathrm{kg} \mathrm{dm}^{-3}$;

Ms - massa do solo seco em estufa a $105^{\circ} \mathrm{C}$, e

$\mathrm{Va}$ - volume do anel, $\mathrm{cm}^{3}$.

A porosidade foi determinada pelo método indireto:

$$
\mathrm{Pt}=\left(1-\frac{\mathrm{Ds}}{\mathrm{Dp}}\right) 100
$$

em que,

$\mathrm{Pt}$ - porosidade total, \%;

Ds - densidade do solo, $\mathrm{kg} \mathrm{dm}^{-3} \mathrm{e}$

$\mathrm{Dp}$ - densidade das partículas $\left(\mathrm{kg} \mathrm{dm}^{-3}\right)$.

O preparo convencional do solo (PC) foi feito com uma passada do arado de discos com objetivo de revolver a leiva do solo e incorporar o resto de cultura e palhada dessecada, depois duas passadas com a grade niveladora para nivelar o solo e destorroá-lo ao ponto de receber as sementes das forrageiras. No plantio direto (PD), as sementes das forrageiras foram depositadas diretamente sobre a palhada dessecada, utilizando-se de uma semeadora-adubadora específica para essa finalidade.

Foram definidas as direções dos eixos cartesianos da malha geoestastística experimental numa área retangular de 40 x $50 \mathrm{~m}$. Os pontos centrais foram alinhados e marcados com auxílio de um teodolito e uma trena para se obter uma malha regular de 5 × $5 \mathrm{~m}$, devidamente referenciada em coordenadas X e Y. A área total para cada sistema de manejo constou de $2.000 \mathrm{~m}^{2}$, com $40 \mathrm{~m}$ no eixo $\mathrm{Y}$ e $50 \mathrm{~m}$ no eixo $\mathrm{X}$, com um total de 99 pontos amostrais.

Foram coletadas amostras indeformadas por meio do método do anel volumétrico de Uhland em dois níveis de profundidade do perfil do solo; 0 a $0,15 \mathrm{~m}$ e 0,15 a $0,30 \mathrm{~m}$, utilizando-se do ponto de cruzamento das coordenadas X, Y como local de amostragem.

As variáveis de produtividade analisadas foram matéria seca total (MS) e índice de área foliar (IAF), avaliados 90 dias após o plantio. Para a determinação da produção de matéria seca, a forrageira foi cortada, em média, a $0,10 \mathrm{~m}$ do solo, em pontos aleatórios da unidade experimental, utilizando-se um de gabarito de $1 \mathrm{~m}^{2}(1 \mathrm{~m}$ x $1 \mathrm{~m})$. O material coletado foi colocado em saco de papel identificado e pesado, e levado em estufa com ventilação forçada, às temperaturas entre 58 e $65^{\circ} \mathrm{C}$, para determinação de sua matéria seca e estimativa da produção de matéria seca total.

O índice de área foliar foi determinado indiretamente com aparelho analisador (ceptômetro) de dossel, marca AccuPAR, modelo LP-80. Em cada unidade experimental, foram realizadas 10 leituras acima e 10 leituras abaixo do dossel. 
Os dados de produção de matéria seca e índice de área foliar foram submetidos à análise exploratória, a partir da qual se obteve a média e a mediana como medidas de tendência central; e a variância e o coeficiente de variação como medidas de dispersão. Também foram determinados os coeficientes de assimetria e curtose para a verificação da posição dos dados em relação à distribuição normal. Os dados foram submetidos ao teste Kolmogorov-Smirnov, para a constatação da aproximação à distribuição normal. Estas análises foram realizadas com os softwares Geostatistics for the Environmental versão 9.0 (GEOSTATISTICS, 1996), Statistical Analysis System (SAS INSTITUTE, 2002) e Sistema para Análises Estatísticas em Genética - SAEG (UNIVERSIDADE FEDERAL DE VIÇOSA - UFV, 2007), considerando um nível de significância igual a $5 \%$.

Para os ajustes dos modelos teóricos dos semivariogramas simples e cruzados, utilizou-se o software $G S+$ em função de seus modelos, foram efetuados pela seleção inicial de: menor soma dos quadrados dos desvios (SQD); maior coeficiente de determinação $\left(\mathrm{R}^{2}\right)$; e maior avaliador da dependência espacial. A decisão final do modelo que representou o ajuste foi realizada pela validação cruzada, assim como para a definição do tamanho da vizinhança que proporcionou a melhor malha de krigagem e/ou cokrigagem. Para cada atributo, foram relacionados o efeito pepita (Co), o alcance (Ao) e o patamar $(\mathrm{Co}+\mathrm{C})$.

O coeficiente de correlação (r) entre tais valores reflete a eficiência do ajuste, dado pela técnica da soma dos quadrados dos desvios, representando a equação de regressão linear em questão. Assim, trabalhando-se na obtenção do número ideal de vizinhos, utilizou-se o software $G S+$ e foram obtidos, por meio da interpolação, os mapas de krigagem ordinária e de cokrigagem, para análise da dependência e da interdependência espacial entre os atributos.

\section{RESULTADOS E DISCUSSÃO}

Os dados relativos à estatística descritiva da produtividade de matéria seca, índice de área foliar, densidade do solo e porosidade total, 90 dias após a semeadura, são apresentados na Tabela 1.

A produção de matéria seca e o índice de área foliar são típicos das regiões tropicais, consequência da estacionalidade das chuvas, além de variações de temperatura e de fotoperíodo. Desta forma, comportamentos semelhantes foram observados para experimentos com capim-brachiaria implantados com plantio direto, sendo $4.020 \mathrm{~kg} \mathrm{ha}^{-1}$ de MS e 4,50 de IAF (ZEFERINO, 2006; LARA, 2007).

Em relação aos valores observados de densidade de solo e porosidade nas duas faixas de profundidade, apresentam-se em condições semelhantes aos de AZEVEDO (2004), avaliando atributos do solo em pastagem degradada sobre um Latossolo Vermelho e um Latossolo Vermelho-Amarelo de textura média, evidenciando uma situação de degradação estrutural na área em estudo, pela compactação do solo. Segundo BORDIN et al. (2005), a compactação do solo pelo uso de práticas inadequadas de manejo das pastagens modifica os atributos físicos do solo, promovendo redução no desenvolvimento do sistema radicular e na produtividade das pastagens.

De acordo com FREDDI (2007), a variabilidade de um atributo pode ser classificada conforme a magnitude do seu coeficiente de variação; sendo assim, é possível observar-se que as variáveis matéria seca e índice de área foliar apresentaram baixa a moderada variabilidade, as baixas registradas no preparo convencional, e a moderada, pelo plantio direto.

Em relação às propriedades físicas do solo, a variabilidade verificada pelo coeficiente de variação apresentou-se baixa para as variáveis Ds2 e Pt2, 7,5 e 14,4\%, respectivamente, e moderada para as variáveis Ds1 e Pt1, 21,0\% em ambas, no preparo convencional. A variabilidade observada no plantio direto foi moderada para a variável Ds1, coeficiente igual a 21,0\%; e baixa para Ds2, Pt1 e Pt2, com coeficientes de 14,7\%, 7,9\% e 14,0\%, respectivamente. Os resultados encontrados são semelhantes aos observados por FREDDI (2007). 
Quanto ao tipo de distribuição, as variáveis MS e IAF apresentaram uma distribuição normal, independentemente do tipo de manejo do solo, ficando o valor dentro da faixa de produtividade média observada por LIMA et al. (2007 e 2009b) e CRUZ et al. (2001).

TABELA 1. Análise descritiva da produtividade de matéria seca e índice de área foliar, da densidade e da porosidade total, 90 dias após a semeadura. Descriptive analysis of dry matter yield and leaf area index, of the density and the total porosity 90 days after sowing.

\begin{tabular}{|c|c|c|c|c|c|c|}
\hline \multirow[b]{2}{*}{ Parâmetros } & \multicolumn{6}{|c|}{ Variáveis $^{1}$} \\
\hline & $\begin{array}{c}\text { MS } \\
\mathrm{kg} \mathrm{ha}^{-1}\end{array}$ & IAF & $\begin{array}{c}\text { Ds1 } \\
\mathrm{kg} \mathrm{dm}^{-3}\end{array}$ & $\begin{array}{c}\text { Ds2 } \\
\mathrm{kg} \mathrm{dm}^{-3}\end{array}$ & $\begin{array}{c}\mathrm{Pt} 1 \\
\mathrm{~m}^{3} \mathrm{~m}^{-3}\end{array}$ & $\begin{array}{c}\mathrm{Pt} 2 \\
\mathrm{~m}^{3} \mathrm{~m}^{-3}\end{array}$ \\
\hline & \multicolumn{6}{|c|}{ Preparo convencional } \\
\hline Média & 4990,08 & 5,21 & 1,43 & 1,57 & 0,399 & 0,319 \\
\hline Mediana & 4964,5 & 5,22 & 1,45 & 1,55 & 0,392 & 0,312 \\
\hline Mínimo & 3881,4 & 4,12 & 1,21 & 1,30 & 0,212 & 0,180 \\
\hline Máximo & 6815,4 & 6,01 & 1,82 & 1,87 & 0,560 & 0,522 \\
\hline Variância & 290566,2 & 0,22 & 0,09 & 0,014 & 0,070 & 0,021 \\
\hline $\mathrm{CV}(\%)$ & 10,8 & 9,0 & 21,0 & 7,5 & 21,0 & 14,4 \\
\hline Assimetria & 0,65 & $-0,206$ & $-0,88$ & $-0,51$ & 0,93 & 0,392 \\
\hline Curtose & 1,51 & $-0,76$ & $-0,030$ & $-0,026$ & 0,550 & 0,521 \\
\hline $\mathrm{D}$ & 0,028 & 0,035 & 0,088 & 0,033 & 0,133 & 0,045 \\
\hline \multirow[t]{2}{*}{$\mathrm{DF}^{(2)}$} & No & No & In & No & In & No \\
\hline & \multicolumn{6}{|c|}{ Plantio direto } \\
\hline Média & 4624,4 & 4,90 & 1,43 & 1,54 & 0,391 & 0,315 \\
\hline Mediana & 4607,3 & 4,89 & 1,45 & 1,50 & 0,388 & 0,311 \\
\hline Mínimo & 2988,5 & 3,33 & 1,20 & 1,25 & 0,230 & 0,174 \\
\hline Máximo & 6505,8 & 6,38 & 1,74 & 1,71 & 0,526 & 0,499 \\
\hline Variância & 643940,9 & 0,40 & 0,09 & 0,034 & 0,001 & 0,002 \\
\hline $\mathrm{CV}(\%)$ & 17,4 & 12,9 & 21,0 & 14,7 & 7,9 & 14,0 \\
\hline Assimetria & 0,26 & $-0,02$ & $-0,72$ & $-0,61$ & 0,69 & 0,303 \\
\hline Curtose & $-0,31$ & 0,34 & $-0,45$ & $-0,26$ & 0,52 & 0,420 \\
\hline $\mathrm{D}$ & 0,041 & 0,039 & 0,111 & 0,043 & 0,212 & 0,038 \\
\hline $\mathrm{DF}^{2}$ & No & No & In & No & In & No \\
\hline
\end{tabular}

${ }^{1}$ MS: produtividade de matéria seca; IAF: Índice de área foliar; Ds: densidade do solo; Pt: porosidade total;

${ }^{2}$ DF: distribuição de frequência, sendo No e In do tipo normal e indeterminada, respectivamente.

Em relação às correlações entre a produtividade de matéria seca, índice de área foliar, densidade do solo porosidade total apresentadas na Tabela 2, é importante ressaltar que a maioria foi baixa, fato que pode ser justificado pelo elevado número de observações. Aquelas que, embora baixas, apresentaram resultados considerados significativos foram as seguintes: MS x Ds1 $(r=$ $\left.-0,201^{*}\right)$, MS x Pt1 $\left(\mathrm{r}=0,189^{*}\right)$ e IAF x Ds1 $\left(\mathrm{r}=0,159^{*}\right)$ observadas no preparo convencional; MS $\mathrm{x} \operatorname{Ds} 1\left(\mathrm{r}=-0,195^{*}\right)$, MS x Pt1 $\left(\mathrm{r}=0,295^{*}\right)$ e IAF x Ds1 $\left(\mathrm{r}=0,307^{*}\right)$ no plantio direto.

Quanto à produtividade de matéria seca e porosidade total, observou-se correlação positiva entre causa e efeito no preparo convencional e no plantio direto, indicando incremento da matéria seca com aumento da porosidade total, na camada de 0,0 a $0,15 \mathrm{~m}$, justificado pelo possível aumento da aeração das raízes da forrageira e, por conseguinte, a absorção radicular, conforme SANTOS et al. (2006).

Em relação às correlações entre as propriedades físicas do solo, vale destacar a alta correlação decrescente entre a densidade do solo e a porosidade na camada de 0,0 a $0,15 \mathrm{~m}$, independentemente do tipo de sistema de manejo do solo, os coeficientes foram $-0,823$ e $-0,772$ para o preparo convencional e plantio direto, respectivamente, AMARO JUNIOR et al. (2007), estudando a variabilidade de atributos físicos do solo em um Latossolo Vermelho encontrou resultados semelhantes a estes, porém vale ressaltar que a comparação depende do teor de umidade do solo (COLET et al., 2009). 
TABELA 2. Matriz de correlação linear simples entre a produtividade de matéria seca, índice de área foliar, densidade do solo e porosidade total. Matrix of simple linear correlation between yield of dry matter, leaf area index, soil density and total porosity.

\begin{tabular}{|c|c|c|c|c|c|c|}
\hline \multirow{2}{*}{ Atributo $^{1}$} & \multicolumn{6}{|c|}{ Coeficiente de Correlação } \\
\hline & MS & IAF & Ds1 & Ds2 & Pt1 & $\mathrm{Pt} 2$ \\
\hline \multicolumn{7}{|c|}{ Preparo convencional } \\
\hline MS & 1 & & & & & \\
\hline IAF & $0,890 *$ & 1 & & & & \\
\hline Ds1 & $-0,201 *$ & $-0,159 *$ & 1 & & & \\
\hline Ds2 & $0,012^{\mathrm{ns}}$ & $0,044^{\mathrm{ns}}$ & $0,152 *$ & 1 & & \\
\hline Pt1 & $0,189 *$ & $0,215^{\mathrm{ns}}$ & $-0,823 *$ & $-0,196 *$ & 1 & \\
\hline Pt2 & $0,195^{\mathrm{ns}}$ & $0,098^{\mathrm{ns}}$ & $-0,068^{\mathrm{ns}}$ & $0,209^{*}$ & $0,059^{\text {ns }}$ & 1 \\
\hline \multicolumn{7}{|c|}{ Plantio direto } \\
\hline MS & 1 & & & & & \\
\hline IAF & $0,798^{*}$ & 1 & & & & \\
\hline Ds1 & $-0,195^{*}$ & $-0,307^{*}$ & 1 & & & \\
\hline Ds2 & $0,021^{\mathrm{ns}}$ & $0,088^{\mathrm{ns}}$ & $0,192 *$ & 1 & & \\
\hline Pt1 & $0,295^{*}$ & $0,171^{\mathrm{ns}}$ & $-0,772 *$ & $-0,289^{*}$ & 1 & \\
\hline Pt2 & $0,166^{\mathrm{ns}}$ & $0,142^{\mathrm{ns}}$ & $-0,145^{\mathrm{ns}}$ & $0,169 *$ & $0,108^{\mathrm{ns}}$ & 1 \\
\hline
\end{tabular}

${ }^{\mathrm{T}}$ MS: produtividade de matéria seca; IAF: índice de área foliar; Ds: densidade do solo; Pt: porosidade total, sendo 1 e 2 nas camadas de 0,0 a 0,15 m e 0,15 a $0,30 \mathrm{~m}$, respectivamente.* significativo a $5 \%$. ${ }^{\text {ns }}$ não significativo.

A análise dos resultados apresentados na Tabela 3 mostra excelentes semivariogramas nos três sistemas de manejo do solo, tanto para produtividade de matéria seca e índice de área foliar, quanto para densidade do solo e porosidade total, sendo que o melhor foi registrado para a densidade na camada de 0,0 a 0,15 m (Ds1) no plantio direto, com coeficiente de determinação espacial de 0,841.

TABELA 3. Parâmetros dos semivariogramas simples ajustados para produtividade de matéria seca, índice de área foliar, densidade do solo e porosidade total. Parameters of the adjusted semivariograms for dry matter yield, leaf area index, soil density and total porosity.

\begin{tabular}{|c|c|c|c|c|c|c|}
\hline \multicolumn{7}{|c|}{ Variável $^{1}$} \\
\hline & MS & IAF & Ds1 & Ds2 & Pt1 & Pt2 \\
\hline & \multicolumn{6}{|c|}{ Preparo convencional } \\
\hline Modelo & Exponencial & Exponencial & Esférico & Exponencial & Gaussiano & Esférico \\
\hline Efeito pepita $\left(\mathrm{C}_{0}\right)$ & $1,27 \times 10^{-6}$ & $1,25 \times 10^{-6}$ & $7,62 \times 10^{-4}$ & $8,0 \times 10^{-4}$ & $3,88 \times 10^{-4}$ & $3,3 \times 10^{-5}$ \\
\hline Patamar $\left(\mathrm{C}+\mathrm{C}_{0}\right)$ & $4,72 \times 10^{-6}$ & $4,60 \times 10^{-6}$ & $2,36 \times 10^{-3}$ & $2,4 \times 10^{-3}$ & $6,0 \times 10^{-3}$ & $1,87 \times 10^{-4}$ \\
\hline Alcance (a) & 22,0 & 21,8 & 25,0 & 13,5 & 22,2 & 16,2 \\
\hline $\mathrm{GDE}^{(4)}$ & Moderada & Moderada & Moderada & Moderada & Forte & Forte \\
\hline $\mathrm{R}^{2}$ & 0,791 & 0,762 & 0,707 & 0,702 & 0,805 & 0,795 \\
\hline \multirow[t]{2}{*}{$\mathrm{SQR}^{(3)}$} & $1,44 \times 10^{-11}$ & $2,24 \times 10^{-12}$ & $4,15 \times 10^{-5}$ & $3,05 \times 10^{-7}$ & $2,99 \times 10^{-10}$ & $3,91 \times 10^{-9}$ \\
\hline & \multicolumn{6}{|c|}{ Plantio direto } \\
\hline Modelo & Exponencial & Exponencial & Esférico & Exponencial & Gaussiano & Esférico \\
\hline Efeito pepita $\left(\mathrm{C}_{0}\right)$ & $3,92 \times 10^{-6}$ & $2,02 \times 10^{-6}$ & $5,00 \times 10^{-4}$ & $6,05 \times 10^{-4}$ & $7,96 \times 10^{-4}$ & $6,29 \times 10^{-5}$ \\
\hline $\operatorname{Patamar}\left(\mathrm{C}+\mathrm{C}_{0}\right)$ & $7,03 \times 10^{-6}$ & $9,22 \times 10^{-6}$ & $7,44 \times 10^{-3}$ & $2,45 \times 10^{-3}$ & $8,08 \times 10^{-3}$ & $1,91 \times 10^{-4}$ \\
\hline Alcance (a) & 24,2 & 21,4 & 19,2 & 16,7 & 18,5 & 17,7 \\
\hline $\mathrm{GDE}^{(4)}$ & Moderada & Forte & Forte & Forte & Forte & Moderada \\
\hline $\mathrm{R}^{2}$ & 0,821 & 0,792 & 0,841 & 0,792 & 0,755 & 0,782 \\
\hline $\mathrm{SQR}^{(3)}$ & $4,44 \times 10^{-11}$ & $1,04 \times 10^{-12}$ & $2,18 \times 10^{-5}$ & $3,95 \times 10^{-7}$ & $5,33 \times 10^{-10}$ & $3,61 \times 10^{-9}$ \\
\hline
\end{tabular}

${ }^{1}$ MS: produtividade de matéria seca; IAF: Índice de área foliar; Ds: densidade do solo; Pt: porosidade total, sendo 1 e 2 nas camadas de 0,0 a $0,15 \mathrm{~m}$ e 0,15 a $0,30 \mathrm{~m} .{ }^{2}$ epp = efeito pepita puro. ${ }^{3} \mathrm{SQR}=$ soma dos quadrados dos resíduos. ${ }^{4} \mathrm{GDE}=$ grau de dependência espacial.

Em relação ao alcance da dependência espacial da produtividade de matéria seca, os valores encontrados foram 22,0 e 24,2 m no preparo convencional e plantio direto, respectivamente; 21,8 e 21,4 $\mathrm{m}$ de alcance da dependência do índice de área foliar nos dois manejos. Os valores extremos observados de alcance para as propriedades físicas do solo foram 13,5 e 25,0 m para as densidades Ds1 e Ds2, respectivamente, ambas no preparo convencional, valores estes semelhantes obtidos por LIMA et al. (2009a) e RAMIREZ-LOPEZ (2008). 
Nas Figuras 1 e 2, mostram-se os mapas das variáveis com valores estimados por krigagem agrupados, após a análise de dependência espacial verificada nos semivariogramas. As variabilidades das densidades do solo e da porosidade total na camada de 0,0 a $0,15 \mathrm{~m}$ e 0,15 a 0,30 m são bastante semelhantes. Observa-se que uma distribuição espacial heterogênea, tanto da produtividade de matéria seca quanto do índice de área foliar são dados que corroboram os estudos de SCHAFFRATH (2006).

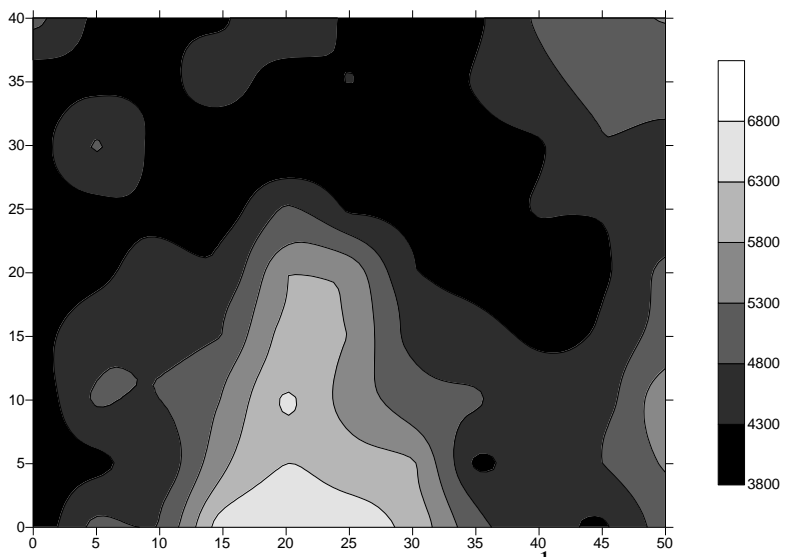

(a) Matéria seca $\left(\mathrm{kg} \mathrm{ha}^{-1}\right)$

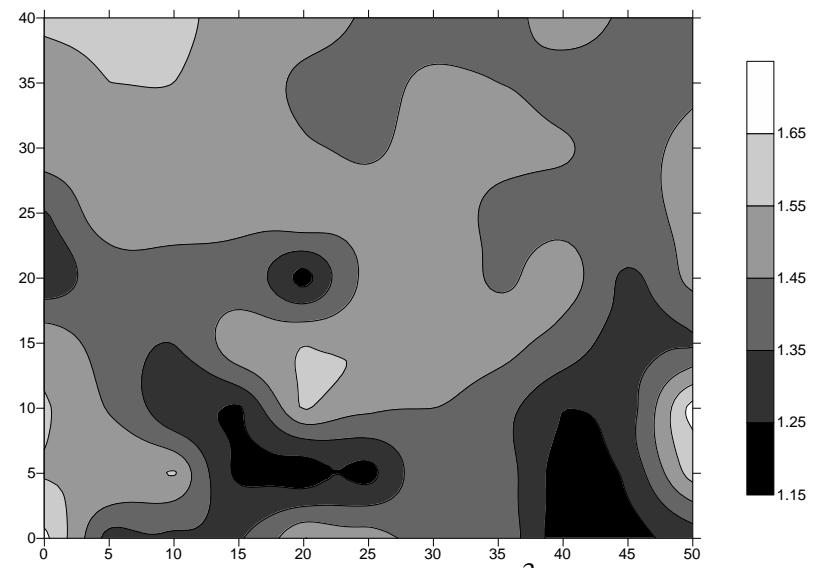

(c) Densidade do solo $\left(\mathrm{kg} \mathrm{dm}^{-3}\right) ; 0,0$ a $0,15 \mathrm{~m}$

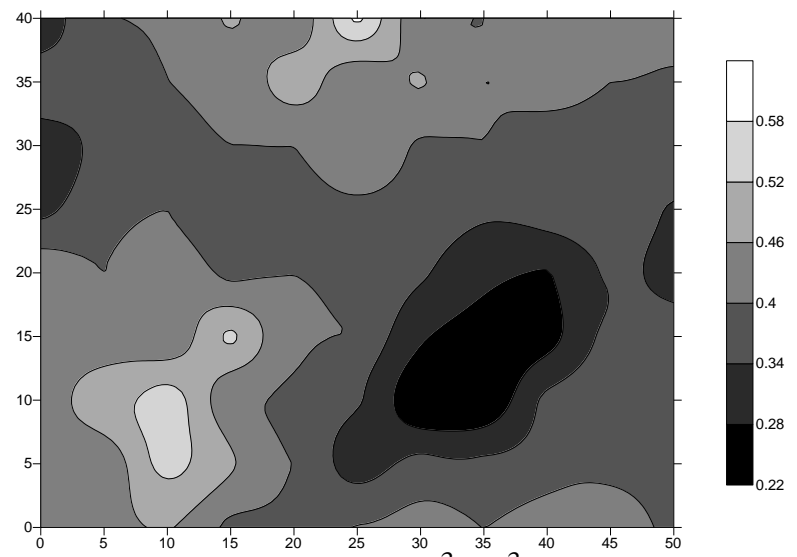

(e) Porosidade total $\left(\mathrm{m}^{3} \mathrm{~m}^{-3}\right) ; 0,0$ a $0,15 \mathrm{~m}$

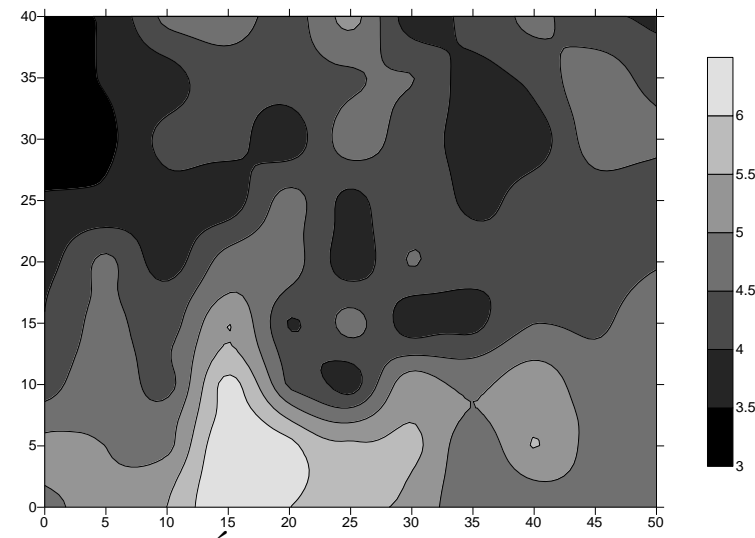

(b) Índice de área foliar

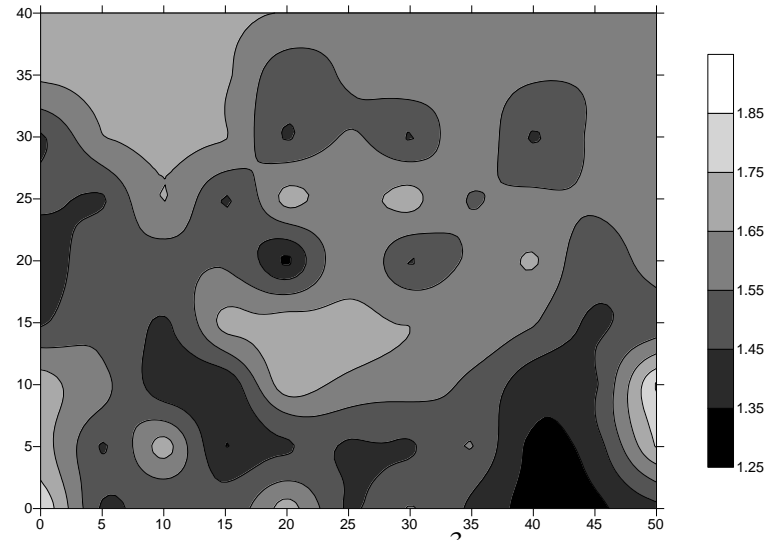

(d) Densidade do solo $\left(\mathrm{kg} \mathrm{dm}^{-3}\right) ; 0,15$ a $0,30 \mathrm{~m}$

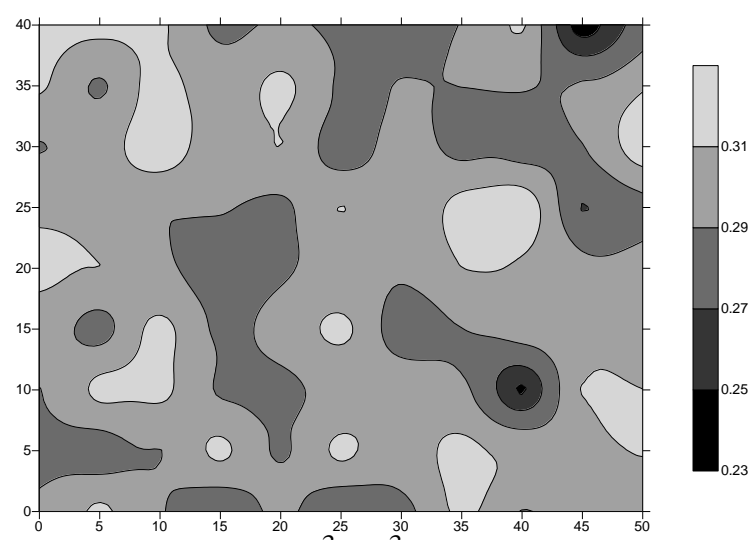

(f) Porosidade total $\left(\mathrm{m}^{3} \mathrm{~m}^{-3}\right) ; 0,15$ a $0,30 \mathrm{~m}$

FIGURA 1. Mapas de krigagem da produtividade de matéria seca da forragem, índice de área foliar, densidade do solo e porosidade total na área preparada com preparo convencional. Kriging maps of the productivity of the forage dry matter, leaf area index, soil density and total porosity in the area prepared with conventional tillage. 


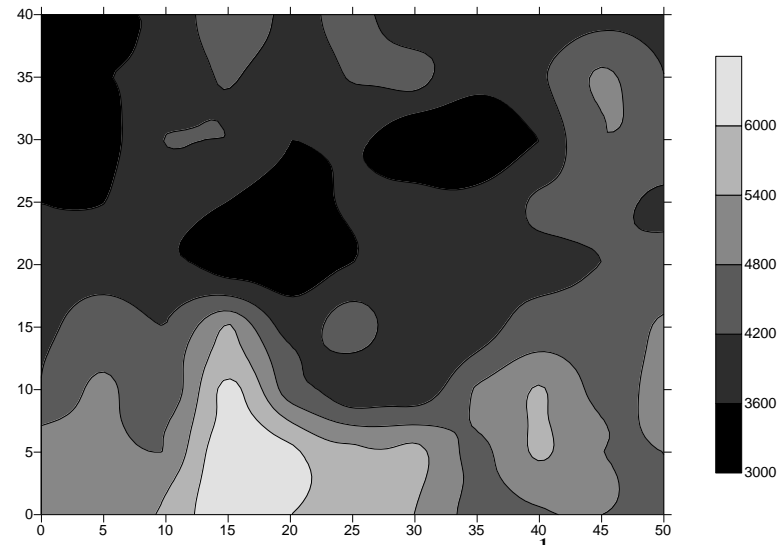

(a) Matéria seca $\left(\mathrm{kg} \mathrm{ha}^{-1}\right)$

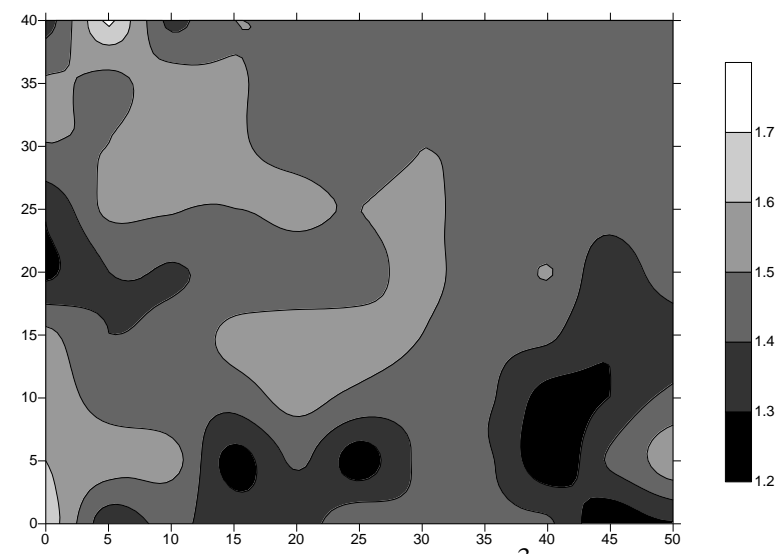

(c) Densidade do solo $\left(\mathrm{kg} \mathrm{dm}^{-3}\right) ; 0,0$ a $0,15 \mathrm{~m}$

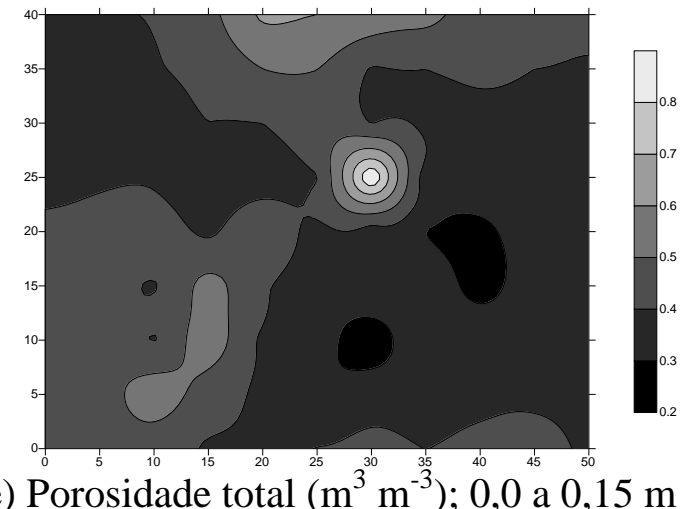

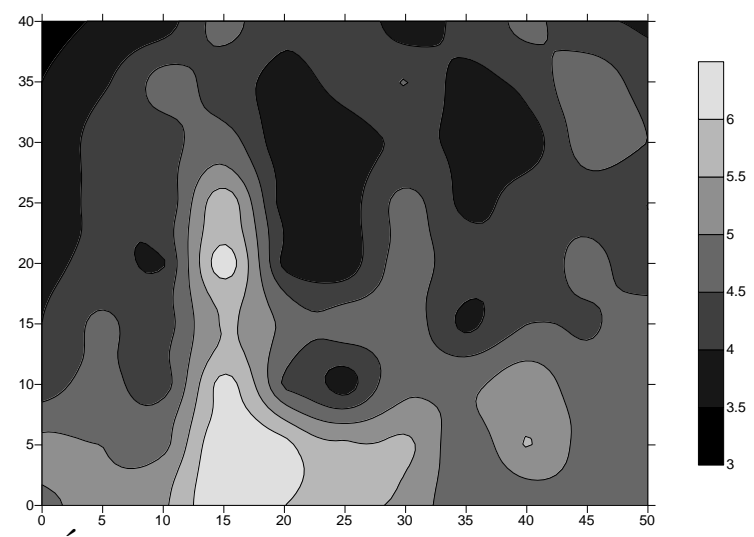

(b) Índice de área foliar

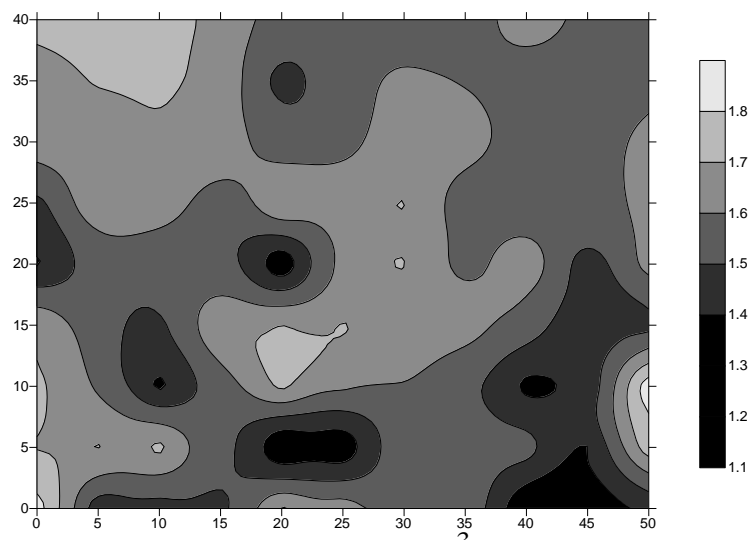

(d) Densidade do solo $\left(\mathrm{kg} \mathrm{dm}^{-3}\right) ; 0,15$ a $0,30 \mathrm{~m}$

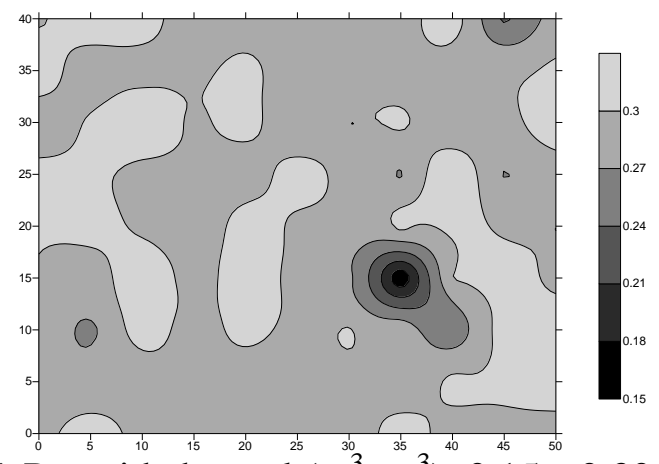

(f) Porosidade total $\left(\mathrm{m}^{3} \mathrm{~m}^{-3}\right) ; 0,15$ a $0,30 \mathrm{~m}$

FIGURA 2. Mapas de krigagem da produtividade de matéria seca da forragem, índice de área foliar, densidade do solo e porosidade total na área de plantio direto. Kriging maps of the productivity of the forage dry matter, leaf area index, soil density and total porosity in the area of tillage.

Observa-se correlação espacial positiva com alcance maior no plantio direto do que no preparo convencional, exceto para porosidade em ambos os manejos de solo. Isto permite constatar que, independentemente da magnitude da variabilidade das propriedades físicas do solo, o plantio direto tende a estender as relações de dependência entre as variáveis, no espaço, em distâncias cada vez maiores. No preparo convencional, por outro lado, o revolvimento do sistema dificulta essa organização no mesmo espaço, de maneira gradativa no tempo, uma vez que, periodicamente, o sistema é revolvido e essa organização é descaracterizada (SCHAFFRATH (2006). 
A análise geoestatística das cokrigagens apresentadas na Tabela 4 indicou que os três melhores semivariogramas cruzados foram para produtividade de matéria seca em função da densidade na camada superficial $(0,0$ a $0,15 \mathrm{~m})$, para os três sistemas de manejo do solo, com coeficientes de determinação espacial de 0,$602 ; 0,592$ e 0,613 no preparo convencional, no cultivo mínimo e plantio direto, respectivamente. Também revelou ajuste esférico para os atributos MS = $\mathrm{f}(\mathrm{Ds} 2), \mathrm{MS}=\mathrm{f}(\mathrm{Pt} 1)$ e $\mathrm{MS}=\mathrm{f}(\mathrm{Pt} 2)$, nos três sistemas de manejo, cujos alcances variaram entre 13,9 e 20,9 m, assim como com elevado grau de dependência espacial para todos, de forma a concordar com SIQUEIRA et al. (2006), que obtiveram, para esse mesmo atributo, pares com correlações variando entre 0,180 e $-0,140$.

TABELA 4. Parâmetros dos semivariogramas cruzados ajustados para produtividade de matéria seca, índice de área foliar, em função da densidade do solo, e porosidade total para cada sistema de manejo do solo. Cross semivariogram parameters adjusted for dry matter yield, leaf area index as a function of soil density and total porosity for each soil management system.

\begin{tabular}{|c|c|c|c|c|}
\hline & \multicolumn{4}{|c|}{ Atributos Cruzados } \\
\hline & $M S=f(D s 1)$ & $M S=f(D s 2)$ & $\mathrm{MS}=\mathrm{f}(\mathrm{Pt} 1)$ & $\mathrm{MS}=\mathrm{f}(\mathrm{Pt} 2)$ \\
\hline & \multicolumn{4}{|c|}{ Preparo convencional } \\
\hline Modelo & Gaussiano & Esférico & Esférico & Esférico \\
\hline Efeito pepita $\left(\mathrm{C}_{0}\right)$ & $-1,00 \times 10^{-1}$ & $1,00 \times 10^{-2}$ & $-1,00 \times 10^{-2}$ & $-1,00 \times 10^{-2}$ \\
\hline Patamar $\left(\mathrm{C}+\mathrm{C}_{0}\right)$ & $-6,22 \times 10^{1}$ & $5,9 \times 10^{1}$ & $2,73 \times 10^{1}$ & $-7,73 \times 10^{1}$ \\
\hline Alcance (a) & 17,3 & 11,7 & 11,7 & 20,1 \\
\hline $\mathrm{GDE}^{4}$ & Forte & Forte & Forte & Forte \\
\hline $\mathrm{R}^{2}$ & 0,602 & 0,217 & 0,556 & 0,472 \\
\hline \multirow[t]{2}{*}{$\mathrm{SQR}^{3}$} & $3,70 \times 10^{3}$ & $5,28 \times 10^{2}$ & $1,33 \times 10^{2}$ & $5,59 \times 10^{2}$ \\
\hline & \multicolumn{4}{|c|}{ Plantio direto } \\
\hline Modelo & Guassiano & Esférico & Esférico & Esférico \\
\hline Efeito pepita $\left(\mathrm{C}_{0}\right)$ & $-1,00 \times 10^{-2}$ & $-1,00 \times 10^{-2}$ & $-1,00 \times 10^{-2}$ & $-1,00 \times 10^{-2}$ \\
\hline $\operatorname{Patamar}\left(\mathrm{C}+\mathrm{C}_{0}\right)$ & $-5,00 \times 10^{1}$ & $4,23 \times 10^{1}$ & $-8,09 \times 10^{1}$ & $-2,96 \times 10^{1}$ \\
\hline Alcance (a) & 19,9 & 13,2 & 11,5 & 20,9 \\
\hline $\mathrm{GDE}^{4}$ & Forte & Forte & Forte & Forte \\
\hline $\mathrm{R}^{2}$ & 0,613 & 0,198 & 0,509 & 0,398 \\
\hline $\mathrm{SQR}^{3}$ & $3,07 \times 10^{3}$ & $4,13 \times 10^{2}$ & $4,24 \times 10^{2}$ & $5,57 \times 10^{2}$ \\
\hline
\end{tabular}

\section{CONCLUSÕES}

A produtividade de matéria seca, o índice de área foliar, a densidade do solo e a porosidade total não variaram aleatoriamente e apresentaram variabilidade dos dados entre baixa e média, independentemente do sistema de manejo do solo.

A correlação linear entre a produtividade de matéria seca e a densidade do solo na camada superficial, ainda que tenha sido baixa, é altamente significativa do ponto de vista espacial, independentemente do manejo do solo.

\section{REFERÊNCIAS}

AMARO FILHO, J.; NEGREIROS, R.F.D.; ASSIS JÚNIOR, R.N.; MOTA, J.C.A. Amostragem e variabilidade espacial de atributos físicos de um Latossolo Vermelho em Mossoró - RN. Revista Brasileira de Ciência do Solo, Viçosa-MG, v.31, n.3, p.415-422, 2007.

AZEVEDO, E. Uso da geoestatística e de recursos de geoprocessamento no diagnóstico da degradação de um solo argiloso sob pastagem no Estado de Mato Grosso. 2004. $141 \mathrm{f}$. Tese (Doutorado em Engenharia Agrícola) - Universidade Estadual de Campinas, Campinas, 2004. 
BORDIN, I.; NEVES, C. S. V. J.; AIDA, F. T.; SOUZA, W. R.; DAVOGLIO JÚNIOR, A. C.; FURLANETO, T. L. R.; TAVARES FILHO, J. Sistema radicular de planta cítrica e atributos físicos do solo em um Latossolo argiloso submetido à escarificação. Ciência Rural, Santa Maria, v.35, n.4, p.820-825, 2005.

COLET, M. J.; SVERZUT, C. B.; WEIRICH NETO, P. H. Alteração em atributos físicos de um solo sob pastagem após escarificação. Ciências Agrotécnicas, Lavras, v.33, n.2, 2009.

CRUZ, J.C.; PEREIRA FILHO, I.A.; RODRIGUES, J.A.S.; FERREIRA, J.F. (Ed.). Produção e utilização de silagem de milho e sorgo. Sete Lagoas: Embrapa Milho e Sorgo, 2001. 544 p.

FOLONI, J.S.S.; CALONEGO, J.C.; LIMA, S.L. Efeito da compactação do solo no desenvolvimento aéreo e radicular de cultivares de milho. Pesquisa Agropecuária Brasileira, Brasília, v.38, p.947-953, 2003.

FREDDI, O.S. Avaliação do intervalo hídrico ótimo em Latossolo Vermelho cultivado com milho. 2007. 105 f. Tese (Doutorado em Produção Vegetal) - Universidade Estadual Paulista, Faculdade de Ciências Agrárias e Veterinárias, Jaboticabal, 2007.

LARA, M.A.S. Respostas morfofisiológicas de cinco cultivares de Brachiaria spp. às variações estacionais da temperatura do ar e do fotoperíodo. 2007. 91 f. Dissertação (Mestrado) - Escola Superior de Agricultura "Luiz de Queiroz”, Piracicaba, 2007.

LIMA, C.G.R.; CARVALHO, M.P.; MELLO, L.M.M.; LIMA, R.C. Correlação linear e espacial entre a produtividade de forragem, a porosidade total e a densidade do solo de Pereira Barreto - SP. Revista Brasileira de Ciência do Solo, v.31, p.1233-1244, 2007.

LIMA, J.S.S.; SATTLER, M.A.; PASSOS, R.R.; OLIVEIRA, P.C.; SOUZA, G.S. Variabilidade espacial de atributos físicos de um Argissolo Vermelho-Amarelo sob pastagem e vegetação secundária em regeneração natural. Engenharia Agrícola, Jaboticabal, v.29, n.2, p.185-195, 2009 b.

LIMA, R.C.; MELLO, L.M.M.; CARVALHO, M.P.; LIMA, C.G.R.; MELLO, A.M. Aspectos lineares e espaciais da correlação entre a produtividade de forragem de milho e a porosidade do solo sob plantio direto. Engenharia Agrícola, Jaboticabal, v.29, n.1, p.40-51, 2009a.

NEIRO, E.S. Propriedades físicas e químicas de um Latossolo Vermelho Distroférrico, sob rotação e sucessão de culturas, sob semeadura direta. 2002. Dissertação (Mestrado em Agronomia) Universidade Estadual de Maringá, Maringá, 2002.

RAMIREZ-LOPEZ, L.; REINA-SANCHEZ, A.; CAMACHO-TAMAYO, J.H. Variabilidad espacial de atributos físicos de un Typic Haplustox de los Llanos Orientales de Colômbia. Engenharia Agrícola, Jaboticabal, v.28, n.1, p.55-63, 2008.

SANTOS, M.L.; CARVALHO, M.P.; RAPASSI, R.M.A.; MURAISHI, C.T.; MALLER, A.; MATOS, F.A. Correlação linear e espacial entre produtividade de milho (Zea mays L.) e atributos físicos de um Latossolo Vermelho distroférrico sob plantio direto do cerrado brasileiro. Acta Scientiarum, Maringá, v.28, n.3, p.313-321, 2006.

SAS INSTITUTE. SAS user's guide: statistics, version 9.1. Cary, 2002.

SCHAFFRATH, V.R. Variabilidade espacial de propriedades físicas do solo e de variáveis de plantas daninhas em sistemas de manejo de solo. 2006. $91 \mathrm{f}$. Tese (Doutorado) - Universidade Estadual de Maringá, Maringá, 2006.

SECCO, D.; DA ROS, C.O.; SECCO, J.K.; FIORIN, J.E. Atributos físicos e produtividade de culturas em um Latossolo Vermelho argiloso sob diferentes sistemas de manejo. Revista Brasileira de Ciência do Solo, v.29, p.407-414, 2005.

SIQUEIRA, G.M. Variabilidade de atributos físicos do solo determinados por métodos diversos. 2006. 163 f. Dissertação (Mestrado) - Instituto Agronômico de Campinas, Campinas, 2006. 
STONE, L.F.; SILVEIRA, P.M. Efeito do sistema de preparo e da rotação de culturas na porosidade e densidade do solo. Revista Brasileira de Ciência do Solo, v.25, p.395-401, 2001.

UNIVERSIDADE FEDERAL DE VIÇOSA - UFV. SAEG: sistema para análises estatísticas, versão 9.1. Viçosa, 2007.

ZEFERINO, C.V. Morfogênese e dinâmica do acúmulo de forragem em pastos de capim-marandu [Brachiaria brizantha (Hoechst. ex A. Rich) cv. Marandu] submetidos a regimes de lotação intermitente por bovinos de corte. 2006. 193 f. Dissertação (Mestrado) - Escola Superior de Agricultura "Luiz de Queiroz”, Piracicaba, 2006. 